\title{
Plummer-Vinson Syndrome with Celiac Disease in A 9 Year Old Boy
}

\section{Marzie Vaghefi $^{1 *}$, Ehsan Sanaee ${ }^{2}$ and Rohallah Edalatkhah ${ }^{3}$}

${ }^{1}$ Resident of Pediatrics, Department of Pediatrics, Shahid sadoughi University of Medical Sciences, Yazd, Iran

${ }^{2}$ Resident of Internal medicine, Department of Internal medicine, Shahid sadoughi University of Medical Sciences, Yazd, Iran

${ }^{3}$ Assistant professor, Department of Pediatrics, Shahid sadoughi University of Medical Sciences, Yazd, Iran

*Corresponding Author: Marzie Vaghefi, Resident of Pediatrics, Department of Pediatrics, Shahid sadoughi University of Medical Sciences, Yazd, Iran.

Received: June 27, 2019; Published: August 17, 2019

\begin{abstract}
Introduction: Plummer-Vinson syndrome is a rare disease that is defined by the classical triad of dysphagia, iron-deficiency anemia and esophageal webs. Even though the syndrome is very rare nowadays, its occurrence in association with celiac disease. Given that the association of Celiac disease with Plummer-Vinson syndrome is uncommon, In this article, We present the case of a patient diagnosed with Plummer-Vinson syndrome and Celiac disease.

Medical history: A 9-year-old boy has been complaining dysphagia over solids about 3 years ago. The patient also suffered from chronic diarrhea and abdominal pain. The patient's pain worsened by eating bread. In a biopsy performed last year from the duodenum, he was diagnosed with celiac disease. In endoscopy, the esophagus web was observed in the upper third of the esophagus.in laboratory, the patient had a hypochromic-microcytic anemia.

Conclusion: Depending on the pathology response and the presence of diagnostic triad(dysphagia, iron-deficiency anemia and esophageal web),The patient was diagnosed with Plummer-Vinson syndrome and Celiac disease, and was treated with balloon dilation and gluten-free diet.
\end{abstract}

Keywords: Plummer-Vinson Syndrome; Celiac Disease; Dysphagia

\section{Introduction}

Plummer-Vinson syndrome is a rare disease that is defined by the classical triad of dysphagia, iron-deficiency anemia and esophageal webs [1]. This disease is associated with atrophic glossitis and angular cheilitis [2]. Plummer-Vinson syndrome, also known as Paterson-Kelly syndrome or sideropenic dysphagia. It is a rare disease, being difficult to determine its prevalence, since in the medical literature only case reports are published [3]. This disease increases the risk of squamous cell carcinoma of the pharynx and esophagus [1]. Even though the syndrome is very rare nowadays, its occurrence in association with celiac disease [1]. Celiac disease is clinical presentation includes the classical malabsorption syndrome, unspecific and extra-intestinal manifestations, and silent celiac disease [4]. The prevalence of Celiac disease in the general population is approximately $1 \%$ and some countries have shown that their prevalence has tripled in a period of 15 years [5].The etiology of the disease is unknown but It is the result of the interaction between gluten and immunological, genetic and environmental fact [6]. In this disease, immune response to gluten causes mucosal injury in the intestine and stimulating the immune system is involved

Citation: Marzie Vaghefi., et al. "Plummer-Vinson Syndrome with Celiac Disease in A 9 Year Old Boy”. Acta Scientific Paediatrics 2.9 (2019): $43-46$. 
in the pathogenesis of the disease [7]. Studies have indicated that the vast majority of patients with CD express HLA-DQ2 or HLADQ8 [8]. The clinical manifestations of CD have a broad spectrum ranging from malabsorption syndrome to asymptomatic disease [9]. The diagnosis of disease is narrow by endoscopy and intestinal biopsy and confirmation of diagnosis is based on the evaluation of the response to gluten-free diet therapy. Using serological tests like Anti-TTG can be useful in diagnosis [10]. The association of this disease with other diseases, such as iron deficiency anemia, herpetiform dermatitis, IGA deficiency, thyroid disorders (Hashimato and Graves), type 1 diabetes and connective tissue disease (SLE, ...) have been proven [10].

Given that the association of Celiac disease with Plummer-Vinson syndrome is uncommon, In this article, We present the case of a patient diagnosed with Plummer-Vinson syndrome and Celiac disease.

\section{Case presentation}

A 9-year-old boy has been complaining dysphagia over solids about 3 years ago. The patient's dysphagia was initially to liquids and then to solids. The patient was referred to and admitted for about a year ago and underwent endoscopy, and he was diagnosed with esophageal web and biopsy taken from his duodenum. The patient was discharged with a recommendation to follow up the pathologic response, but the follow up was not performed by the patient. The patient also suffered from chronic diarrhea and abdominal pain in addition to complaining of a lack of improvement in dysphagia. The patient's pain worsened by eating bread. Following the pathologic response of the patient, it was determined that the patient was diagnosed with celiac disease. In the tests performed for the patient, the level of Anti-TTG was high, but the level of total IgA was normal (Anti-TTG=89).

\section{Physical examination}

In the head and neck of the patient, angular cheilitis was observed. Also, The conjunctiva was pale. Hypopigmented skin rash was seen in the patient's face, which seemed to be Pityriasis alba. in abdoman examination, the abdomen was soft, did not have a guarding or distention, had a slight tenderness in the periumbilical area.

\section{Laboratory}

In Cell Blood Count (CBC), The patient had leukocytosis $(\mathrm{WBC}=12400)$. Also,the patient had a hypochromic-microcytic anemia $(\mathrm{HG}=8.8, \mathrm{MCV}=62.38, \mathrm{MCH}=17.15)$.

\section{Investigation and treatment}

The patient was again subjected to endoscopy and the esophagus web was observed in the upper third of the esophagus (figure 1). For the patient the balloon dilation was performed. Then, the patient was diagnosed with Plummer-Vinson syndrome and celiac disease (figure 2), and was treated with gluten-free diet, and discharged with a recommendation to do the follow up.

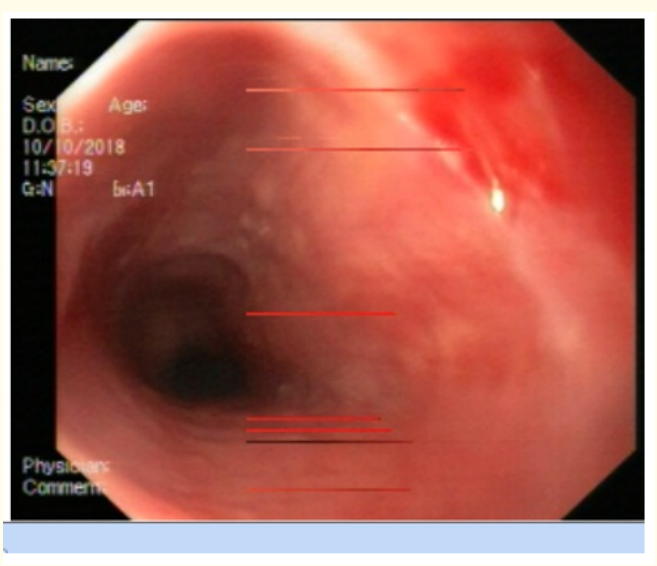

Figure 1: Web of esophagus in endoscopy.

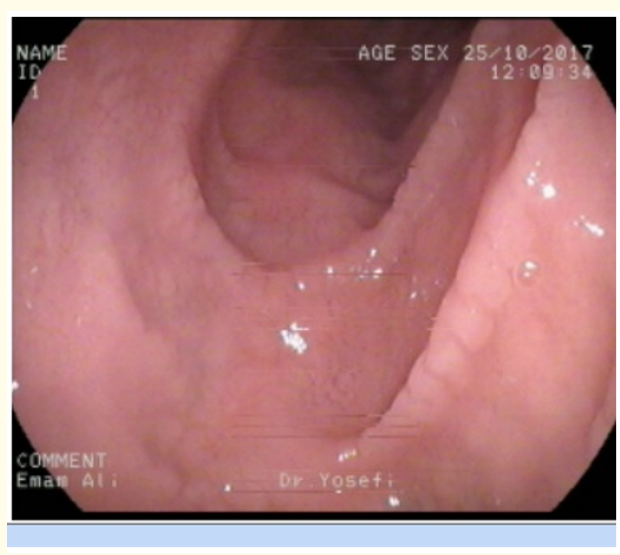

Figure 2: (duodenum). 


\section{Discussion and Conclusion}

Plummer-Vinson syndrome is a rare disease; So that in the medical literature only case reports are published [3]. The combined of Plummer-Vinson syndrome with Celiac disease has also been reported rarely in several studies in recent years [1]. The first report was published by Dickey and Mc Connell in 1999 [12]. They describe two patients with Plummer-Vinson syndrome whose iron deficiency anemia was due to celiac disease. They presented with dysphagia 13 and 9 years, respectively, before celiac disease was diagnosed [12]. In other study in 2010, Leila Mnif and., et al. introduced a case of Plummer-Vinson syndrome and Celiac disease. The patient in their study was A 28-year-old white woman was referred to endoscopic department for dilation of upper esophageal web [13].

Postcricoid carcinoma as complications of unrecognized celiac disease is common.In two study,two patients with combined of Plummer-Vinson syndrome with Postcricoid carcinoma as complications of unrecognized Celiac disease as case-report Introduced $[14,15]$.

In our report, in pathology, Sections show poorly oriented duodenal mucosa. the preserved villi show blunting and increased number of intraepithelial lymphocytes (about 45l lymphocytes/100 enterocytes). The lamina propria is infiltrate by some lymphoplasma cells and few eosinophil. the findings are characteristic of GSE (MARSH III a) and clinical correlation is recommended (figure 3 and figure 4).

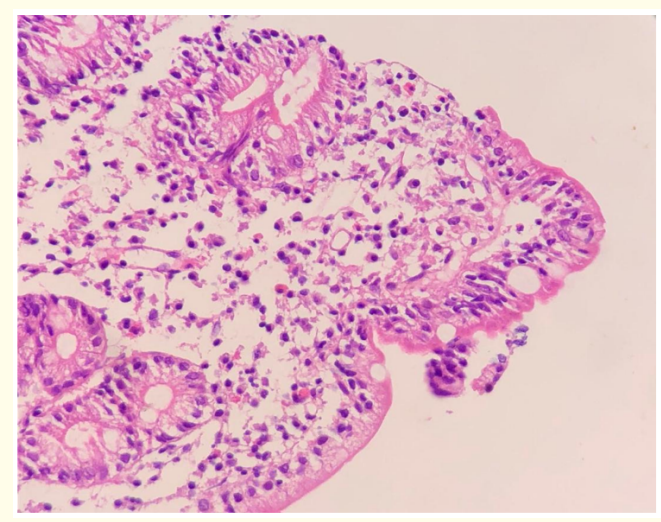

Figure 3: $\mathrm{H}$ and E stain- (duodenal biopsy).

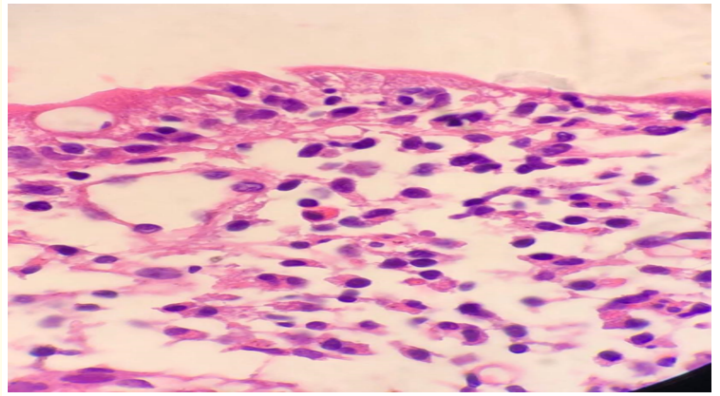

Figure 4: $\mathrm{H}$ and E stain- (duodenal biopsy).

Depending on the pathology response and the presence of diagnostic triad (dysphagia, iron-deficiency anemia and esophageal web), The patient was diagnosed with Plummer-Vinson syndrome and Celiac disease, and was treated with balloon dilation and gluten-free diet.

\section{Conflict of Interest:}

There is no conflict of interest regarding the publication of this paper.

\section{Bibliography}

1. Novacek G. "Plummer-Vinson syndrome". Orphanet Journal of Rare Diseases 1 (2006): 36.

2. Demirci F., et al. "Plummer-Vinson syndrome and dilation therapy: a report of two cases". Turkish Journal of Gastroenterology 16.4 (2005): 224-227.

3. Dias IS., et al. "Plummer-Vinson syndrome: a case report". Revista do Colégio Brasileiro de Cirurgiões 40.1 (2013): 81-82.

4. Kagnoff M. "Overview and Pathogenesis of Celiac Disease". Gastroenterology 128 (2005): s10-s8.

5. JP Burger., et al. "Rising incidence of celiac disease in the Netherlands: an analysis of temporary trends from 1995 to 2010". Scandinavian Journal of Gastroenterology 49.8 (2014): 933941.

6. Kilmartin C., et al. "Avenin fails to induce Th1 response in coeliac tissue following in vitro culture". Gut 52.1 (2003): 47-52.

7. James MW and Scott BB. "Coeliac Disease: The Cause of the Various Associated Disorders?". European Journal of Gastroenterology and Hepatology 13.9 (2001): 1119-1112. 
8. Sollid LM and Lie BA. "Celiac disease genetics: current concepts and practical applications". Clinical Gastroenterology and Hepatology 3.9 (2005): 843-851.

9. Di Sabatino A and Corazza G. "Coeliac disease". Lancet 373.9673 (2009): 1480-1494.

10. Fasano A and Catassi C. "Current approaches to diagnosis and treatment of celiac disease: an devolving spectrum". Gastroenterology 120.3 (2001): 636-651.

11. Green PH. "The many faces of celiac disease: clinical presentation of celiac disease in the adult population". Gastroenterology 128.4-1 (2005): S74-S78.

12. Dickey $\mathrm{W}$ and McConnell B. "Celiac disease presenting as the Paterson-Brown Kelly (Plummer-Vinson) syndrome”. The American Journal of Gastroenterology 94.2 (1999): 527-529.

13. Mnif L., et al. "Celiac disease presenting as Plummer-Vinson syndrome”. La Tunisie Medicale 88.11 (2010): 858.

14. Rashid Z., et al. "Plummer-Vinson syndrome and postcricoid carcinoma: late complications of unrecognized celiac disease". The American Journal of Gastroenterology 94.7 (1999): 1991.

15. Jessner W., et al. "Plummer-Vinson syndrome associated with celiac disease and complicated by postcricoid carcinoma and carcinoma of the tongue". The American Journal of Gastroenterology 98.5 (2003): 1208-1209.

Volume 2 Issue 9 September 2019

(C) All rights are reserved by Marzie Vaghefi., et al. 